\title{
Resolution of immune activation defines nonpathogenic SIV infection
}

\author{
Olivier Manches and Nina Bhardwaj
}

New York University Langone Medical Center and New York University Cancer Institute, New York, New York, USA.

\begin{abstract}
Natural nonhuman primate hosts of SIV do not succumb to AIDS despite significant viral replication, a phenomenon attributed to reduced levels of chronic and deleterious "immune activation." Two studies in this issue of the JCI, by Bosinger et al. and Jacquelin et al., now show that SIV induces vigorous immune activation and upregulation of IFN-stimulated genes in both natural and susceptible hosts, but strikingly, the responses resolve only in the former (see the related articles beginning on pages 3556 and 3544, respectively). Thus, natural hosts for SIV actively engage mechanisms to abort sustained immune activation and its associated harmful effects.
\end{abstract}

Chronic, generalized immune activation is believed to advance the pathogenesis of progressive HIV and SIV infection $(1,2)$. The phenomenon, observed in humans and in susceptible strains of monkeys (e.g., rhesus macaques [RMs]), is associated with ineffective control of virus replication; accelerated apoptosis and turnover of $\mathrm{T}$ and B lymphocytes; increased levels of T cells expressing markers of activation and proliferation (e.g., CD38, antigen identified by monoclonal antibody Ki-67 [MKI67]); and elevated serum levels of proinflammatory cytokines including type I IFN (reviewed in ref. 2). Of significance, the level of immune activation in the early phase of HIV-1 infection is prognostic of disease outcome. In contrast, SIV infection in natural hosts (e.g., African green monkeys [AGMs] and sooty mangabeys [SMs]) is nonpathogenic despite persistent viral replication, possibly because they do not develop features of chronic immune activation (3).

Although the etiology of immune activation is multifactorial, it is generally thought that chronic high levels of type I IFN and proinflammatory cytokines are central to its maintenance. Type I IFN, in particular, besides upregulating IFN-stimulated genes (ISGs) and inducing an antiviral state, contributes to generalized activation of lymphocytes and NK cells, exhaustion and apoptosis of $\mathrm{T}$ cells, defects in thymopoiesis, maturation of immature DCs, and aberrant hematopoietic stem cell pro-

Conflict of interest: The authors have declared that no conflict of interest exists.

Citation for this article: J. Clin. Invest. 119:3512-3515 (2009). doi:10.1172/JCI41509. liferation (reviewed in ref. 4). Diminished production of IFN- $\alpha$ by plasmacytoid DCs (pDCs) due to mutations in the transactivation domain of IFN regulatory factor 7 (IRF7) has been postulated to account for the nonpathogenicity of infection in natural hosts (5). However, natural hosts mount anti-SIV $\mathrm{T}$ cell responses not dissimilar to RMs (6), maintain normal CD4 ${ }^{+}$ $\mathrm{T}$ cell homeostasis in blood, and have evidence of strong type I IFN responses that subsequently resolve (7). Thus, it remains unresolved whether the lack of sustained immune activation is due to a general attenuated response to infection, or to induction of regulatory mechanisms that suppress immune responses generated during the acute infection.

\section{Evidence of immune activation in natural hosts of SIV}

In this issue of the JCI, Bosinger et al. (8) and Jacquelin et al. (9) evaluate the role of declining immune activation in the control of progressive disease in natural hosts of SIV. The transcriptional host response to SIV infection from the initial infection to the chronic phase was measured in SMs and AGMs (infected with SIVsmm and SIVagm, respectively) and compared with that in SIVmac239-infected RMs using high-throughput microarrays. Evaluating whole blood, lymph nodes, or $\mathrm{CD}^{+} \mathrm{T}$ cells from each source, both studies documented a strong antiviral response early after infection in all species, characterized by upregulation of numerous transcripts associated with innate and adaptive immune responses (e.g., pattern recognition receptors), chemokines, antiviral restriction fac- tors, and a large number of ISGs. Secreted IFN- $\alpha$ was detectable in plasma at the time of peak viral load in AGMs. Thus, the acute phase of pathogenic and nonpathogenic SIV infection is characterized by an integrated, strong antiviral response with clear evidence of intact type I IFN production, contrary to published reports (5) but consistent with recent studies in AGMs (7). However, the transcriptional profile in the chronic phase of infection differed significantly, the expression of the highly upregulated ISGs being downregulated to baseline in AGMs and SMs. In RMs, chronic infection was characterized by not only persistent expression of ISGs, but increased expression of markers associated with cell activation and proliferation (e.g., MKI67, CD38, chemokines, cell cycling genes) and $\mathrm{CD}^{+} \mathrm{T}$ cell exhaustion (e.g., lymphocyteactivation gene 3 [LAG3], T cell immunoglobulin mucin 3 [TIM3]). Thus, while all species demonstrated robust innate and adaptive immune responses during the acute phase of SIV infection, only AGMs and SMs downmodulated their responses during chronic infection (Figure 1).

\section{Factors underlying resolution of immune activation during chronic infection}

Authors of both studies $(8,9)$ invoke the induction of regulatory mechanisms to explain the resolution of immune activation in SIV-infected natural hosts versus SIV-infected RMs. For example, AGMs induce IFN and ISGs more rapidly than RMs following infection, perhaps effectively containing viral replication (7-9). Acutely, SIV-infected SMs had higher levels of the tryptophan-depleting enzyme indoleamine 2,3 dioxygenase (IDO) than RMs (although this was not the case in AGMs), as well as ADAR (an adenosine deaminase that suppresses ISG expression), host restriction factors, and antiapoptotic regulators (Figure 1). One month after infection, the expression of defensins, cysteine-rich cationic peptides with antimicrobial activity, was augmented. During the transition 
A Nonpathogenic SIV infection of African green monkeys and sootey mangabeys

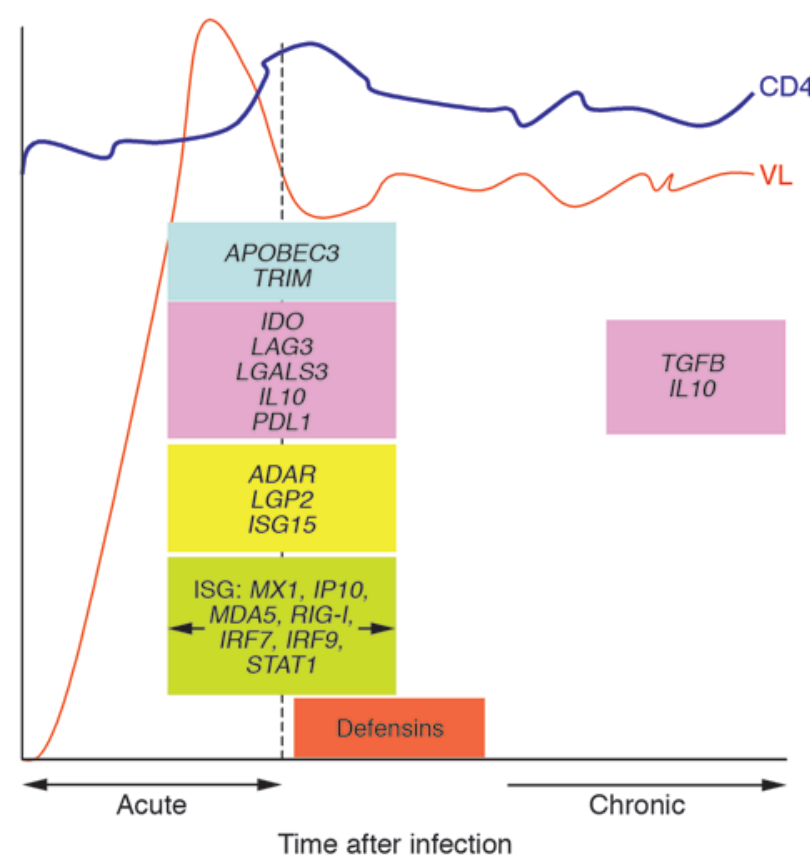

B Pathogenic SIV infection of rhesus macaques

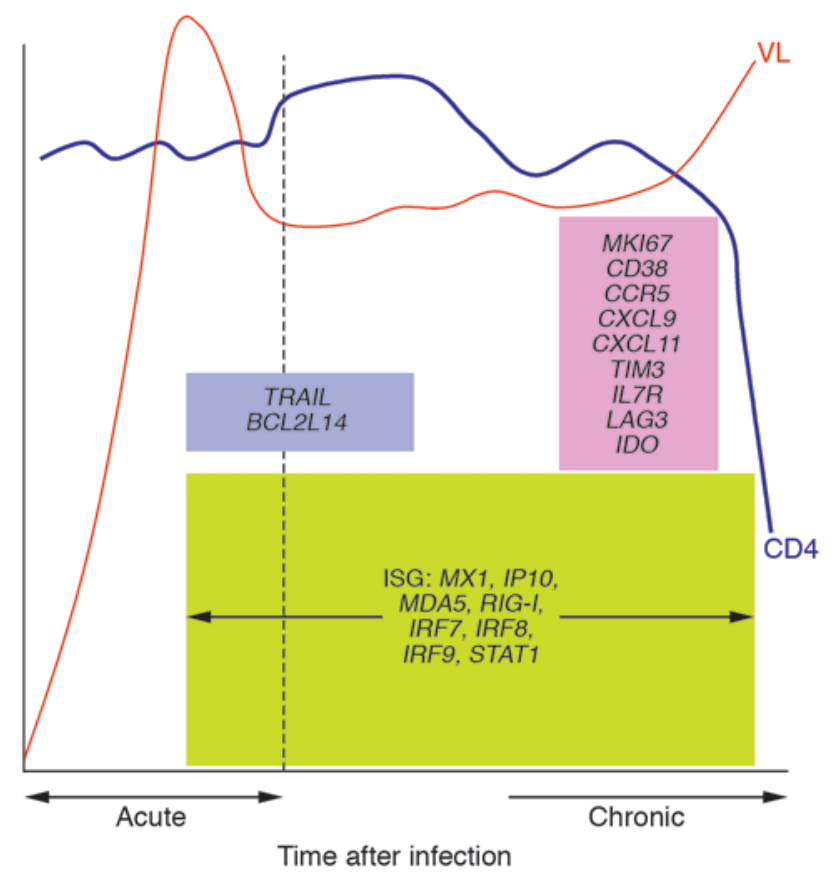

Figure 1

Transcriptional profile of pathogenic and nonpathogenic SIV infections. (A) Nonpathogenic SIV infections in SMs and AGMs are characterized by sustained CD4+ T cell counts and controlled viremia (viral load [VL]). There is early upregulation of transcripts for viral restriction factors (blue), of genes associated with dampening of T cell activation (pink), of antimicrobial defensins (red), and of ISGs (green). However, the expression of ISGs resolves rapidly, possibly due to upregulation of suppressors of the IFN response (yellow). Transcripts for the immune-dampening genes TGFB and IL10 are upregulated in chronic infection (pink). Myxovirus influenza virus resistance protein 1 (MX1) and IP10 are antiviral factors. Melanoma differentiation-associated protein 5 (MDA5) and retinoic acid-inducible gene I (RIG-I) are intracellular sensors of RNA viruses. IRF7, IFN regulatory factor 7. (B) In contrast, SIV infection of RMs is characterized by eventual loss of CD4+ $\mathrm{T}$ cells and corresponding loss of control of viremia. RMs have chronic expression of ISGs, accompanied by enhanced expression of proapoptotic factors (purple), as well as markers of $T$ cell activation and exhaustion and of inflammation (pink). Antiviral host restriction factors: apolipoprotein B mRNA-editing enzyme, catalytic polypeptide-like 3 (APOBEC3), T cell receptor-interacting molecule (TRIM) family members. T cell-associated inhibitory factors: LAG3, IL10, programmed cell death ligand 1 (PDL1), soluble galactoside-binding lectin 3 ( $L G A L S 3$; also known as galectin-3, a proapoptotic ISG), and IDO. Suppressors of IFN: adenosine deaminase, RNA-specific $(A D A R)$, laboratory of genetics and physiology 2 (LGP2), and IFN-stimulated gene 15 (ISG15). Proapoptotic molecules: TRAIL and B cell CLL/lymphoma 2-like 14 (BCL2L14). Markers of T cell activation and exhaustion: MKI67 (marker of proliferation), CD38, CCR5, CXCL9, and CXCL11, TIM3, IL7-receptor (IL7R), and LAG3.

to the chronic phase, there was increased expression of the immune-suppressive cytokines IL-10 and TGF- $\beta$ in AGMs (9), as previously noted (10), as well as of LAG3, a marker associated with $\mathrm{CD}^{+}{ }^{+}$Tregs. In contrast, the sustained immune activation of RMs was accompanied by higher levels of

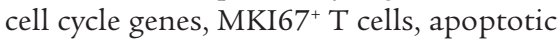
genes (e.g., TNF-related apoptosis-inducing ligand [TRAIL]), and T cell-homing chemokines in the LN, suggesting local sequestration and perpetuation of inflammatory responses. Thus, mechanisms that limit susceptibility to virus infection (i.e., rapid ISG and viral restriction factor production) and immunosuppressive responses (e.g., IL-10, TGF- $\beta$, Tregs) may be critical in protecting natural hosts against pathogenic infection.
Both studies rule out desensitization of IFN- $\alpha$-producing cells to SIV or IFN- $\alpha$ itself as a cause of reduced immune activation $(8,9)$. Circulating cells from chronically infected SMs or AGMs remained sensitive to IFN- $\alpha$ and upregulated ISGs, even at low doses of IFN- $\alpha$. Furthermore, Jacquelin et al. (9) showed that cells from uninfected AGMs and RMs, as well as chronically infected AGMs, were able to secrete IFN- $\alpha$ upon in vitro stimulation by autologous virus. Nevertheless, it is possible that pDCs in inflammatory sites such as LN, where viral loads are high, could become selectively desensitized, which would explain the persistent viral replication in natural hosts despite the absence of ISG induction. Alternatively, subtle differences in the amplitude and quality of the acute immune response may translate into widely different outcomes of infection. Jacquelin et al. found that IFN- $\alpha$ sustains a feed-forward loop enhancing secondary IFN- $\alpha$ responses, likely through upregulation of IRF7 or IRF8, expression of which is sustained in LNs of RMs. Lower expression of CCR5, the SIV coreceptor, on CD4 ${ }^{+} \mathrm{T}$ cells of natural hosts (11) and reduced expression of T cell-homing chemokines (IFN-inducible protein 10 [IP10], CCL5, CXCL9, CXCL11; ref. 8) might reduce trafficking of activated $\mathrm{CD}^{+} \mathrm{CCR}^{+} \mathrm{T}$ cells to inflamed tissues. Moreover, reduction of CD3-TCR complex levels in natural hosts by SIV Nef may further reduce responsiveness of $T$ cells (2). Finally, rapid infiltration of programmed cell death 1-express- 


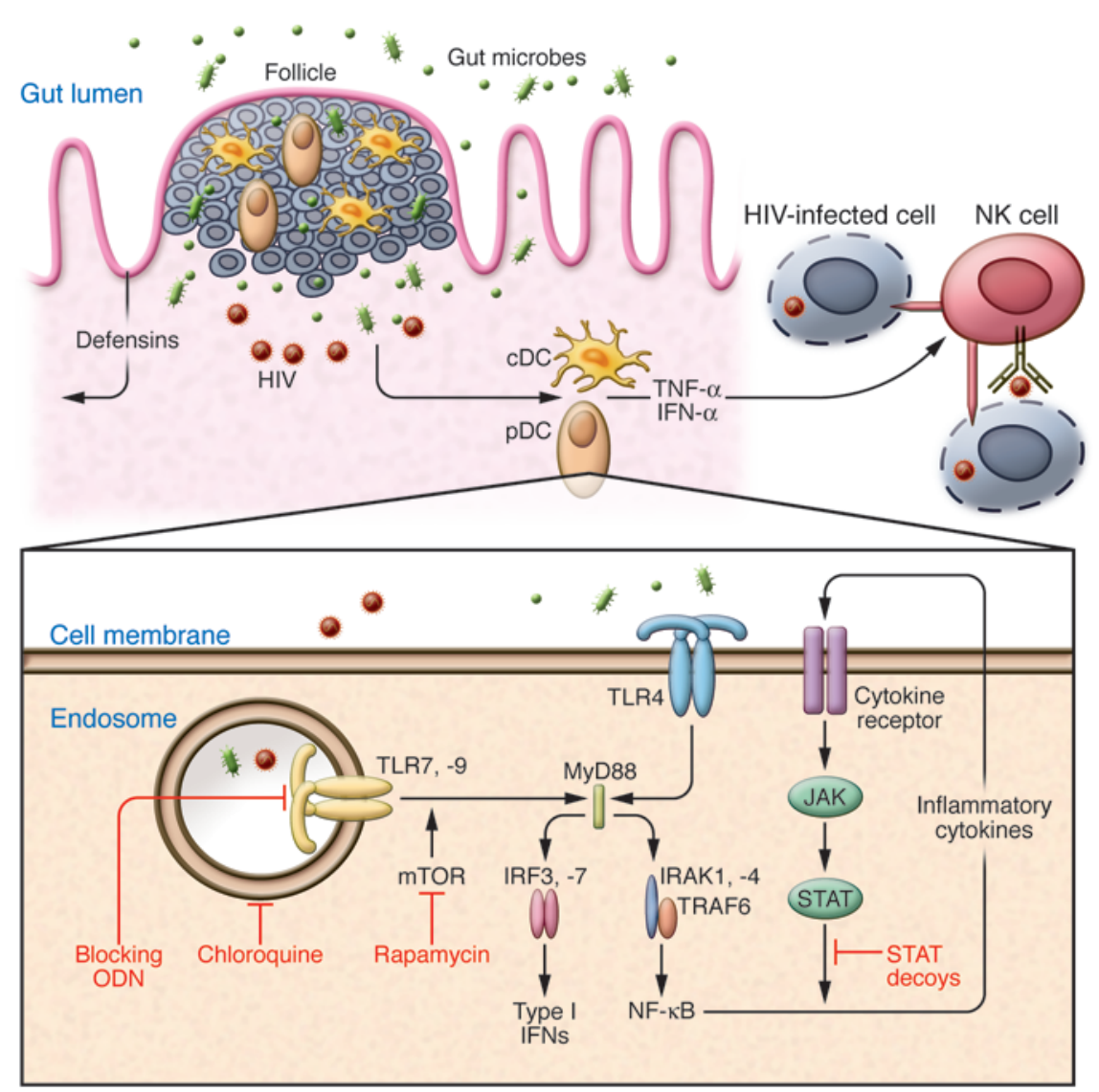

ing (PD-1-expressing) CD4- $\left(\mathrm{CD}^{+}\right) \mathrm{T}$ cells in LNs of SIV-infected SMs may also downregulate immune activation (12).

\section{Lessons from natural hosts for HIV vaccine design and therapy}

Natural hosts for SIV, unlike RMs and HIV-infected humans, seem to rely less on adaptive immune responses to control disease pathogenesis (13). Nevertheless, the natural host models inform us that stimulation of specific protective innate (in addition to adaptive) immune responses early, combined with suppression of sustained systemic immune activation, may be key to preventing disease progression in HIVinfected humans. This could involve targeting APCs, e.g., pDCs, primary producers of type I IFN with the inherent ability to present viral antigens to T cells. HIV induction of IFNs by pDCs, which is TLR mediated (14), is accompanied by IDO-dependent induction of Tregs, which limit $\mathrm{T}$ cell proliferation and bystander activation of DCs (15). Adjuvants that take advantage of this property (e.g., synthetic TLR agonists) could be tested. On the flip side, TLR antagonists may limit chronic, HIV- induced IFN production or TLR-mediated stimulation of gut or blood DCs by gut microflora or via microbial translocation, respectively (Figure 2). Nevertheless, the postulated central role of type I IFN in perpetuating immune activation can now be directly tested in chronically infected RMs using neutralizing antibodies or pharmacological inhibitors of the TLR/IFN pathway (Figure 2) (16). Likewise, chronically infected AGMs or SMs could be administered type I IFNs to determine whether infection becomes pathogenic in an environment of high ISG expression.

Certain NK cell receptors are associated with slower HIV- $1^{+}$disease progression: coexpression of HLA-Bw4 alleles and KIR3DL1 allotypes confers a protective advantage (17). NK cells may have direct cytolytic activity upon virus-infected cells or be directed through antibody-dependent cell cytotoxicity (ADCC) to eliminate target cells. The in vivo efficacy of the HIV-1-neutralizing antibody b12 is largely mediated by its Fc component, implicating ADCC in its effectiveness (18). As NK cell activity may be higher in uninfected or infected SMs versus RMs (19), study of the NK cell func-

\section{Figure 2}

Possible strategies for manipulation of innate immunity in pathogenic infections. Chronic immune activation is a multifactorial process driven by many cell types, including conventional DCs (cDCs), pDCs, T cells, or NK cells. DCs recognizing HIV or microbial components through TLRs or other pattern recognition receptors secrete IFN- $\alpha$ and inflammatory cytokines, inducing activation of other cell types (e.g., NK cells). Activated NK cells can lyse virally infected cells directly or through ADCC. Inhibitors of the TLR pathway or of cytokine signaling can be used to tune down the inflammatory response. For example, chloroquine (14) and short oligonucleotides (ODNs) block activation of endosomal TLR. Rapamycin inhibits mammalian target of rapamycin (mTOR) signaling, necessary for recruitment of MyD88 to TLRs (16). STAT decoys can inhibit the transcriptional program induced in target cells by inflammatory cytokines. Synthetic mimetics of defensins, peptides with antimicrobial activity, could be used to protect gut epithelium from bacterial invasion and enhance anti-HIV immune responses. TRAF6, TNF receptor-associated factor 6; IRAK1, IL-1 receptor-associated kinase 1.

tional repertoire, $\mathrm{FcR}$ polymorphisms, and the contribution of the breadth and neutralizing capacity of antibodies toward SIV in natural hosts is necessary. Members of the innate immune system with otherwise compromised function in pathogenic hosts ( $\gamma \delta \mathrm{T}$ cells) also require further evaluation.

The altered balance of Th and regulatory $\mathrm{T}$ cell subsets in chronic HIV infection contributes to disease progression, Th17 being crucial for controlling microbial dissemination in the gastrointestinal tract and Tregs being important for preventing excessive immune activation and gut damage. Given that microbial translocation does not occur in natural nonhuman primate hosts, these mucosal defense pathways are likely to be intact (20). The pathways for Th17 and Treg differentiation are complex and interrelated, but experimental manipulation of their differentiation is possible (21).

Study of natural hosts of SIV has yielded important new information regarding resistance to pathogenesis. Further studies are now warranted to yield an integrated picture of the immune regulation occurring in natural hosts, but one will probably need to target multiple points to counteract the 
damaging immune activation triggered by HIV infection in humans (Figure 2).

\section{Acknowledgments}

Research in our laboratory that was cited in this commentary was supported by NIH grants AI044628, AI061684, and P01 AI057127.

Address correspondence to: Nina Bhardwaj, The New York University Langone Medical Center, Smilow Research Building, 522 First Avenue, Room 1303, New York, New York 10016, USA. Phone: (212) 2635814; Fax: (212) 263-6729; E-mail: Nina. Bhardwaj@med.nyu.edu.

1. Hazenberg, M.D., et al. 2003. Persistent immune activation in HIV-1 infection is associated with progression to AIDS. AIDS. 17:1881-1888.

2. Paiardini, M., Pandrea, I., Apetrei, C., and Silvestri, G. 2009. Lessons learned from the natural hosts of HIV-related viruses. Annu. Rev. Med. 60:485-495.

3. Silvestri, G., et al. 2005. Divergent host responses during primary simian immunodeficiency virus SIVsm infection of natural sooty mangabey and nonnatural rhesus macaque hosts. J. Virol. 79:4043-4054.

4. Theofilopoulos, A.N., Baccala, R., Beutler, B., and Kono, D.H. 2005. Type I interferons (alpha/beta) in immunity and autoimmunity. Annu. Rev. Immunol. 23:307-336.

5. Mandl, J.N., et al. 2008. Divergent TLR7 and TLR9 signaling and type I interferon production distinguish pathogenic and nonpathogenic AIDS virus infections. Nat. Med. 14:1077-1087.

6. Wang, Z., Metcalf, B., Ribeiro, R.M., McClure, H., and Kaur, A. 2006. Th-1-type cytotoxic CD8+ T-lymphocyte responses to simian immunodeficiency virus (SIV) are a consistent feature of natural SIV infection in sooty mangabeys. J. Virol. 80:2771-2783.

7. Lederer, S., et al. 2009. Transcriptional profiling in pathogenic and non-pathogenic SIV infections reveals significant distinctions in kinetics and tissue compartmentalization. PLoS Pathog. 5:e1000296.

8. Bosinger, S.E., et al. 2009. Global genomic analysis reveals rapid control of a robust innate response in SIV-infected sooty mangabeys. J. Clin. Invest. 119:3556-3572.

9. Jacquelin, B., et al. 2009. Nonpathogenic SIV infection of African green monkeys induces a strong but rapidly controlled type I IFN response. J. Clin. Invest. 119:3544-3555.

10. Kornfeld, C., et al. 2005. Antiinflammatory profiles during primary SIV infection in African green monkeys are associated with protection against AIDS. J. Clin. Invest. 115:1082-1091.

11. Pandrea, I., et al. 2007. Paucity of CD4+CCR5+ T cells is a typical feature of natural SIV hosts. Blood. 109:1069-1076.

12. Estes, J.D., et al. 2008. Early resolution of acute immune activation and induction of PD- 1 in SIV-infected sooty mangabeys distinguishes nonpathogenic from pathogenic infection in rhesus macaques. J. Immunol. 180:6798-6807.

13. Sodora, D.L., and Silvestri, G. 2008. Immune activation and AIDS pathogenesis. AIDS. 22:439-446.

14. Beignon, A.S., et al. 2005. Endocytosis of HIV-1 activates plasmacytoid dendritic cells via Tolllike receptor-viral RNA interactions. J. Clin. Invest. 115:3265-3275.

15. Manches, O., et al. 2008. HIV-activated human plasmacytoid DCs induce Tregs through an indoleamine 2,3-dioxygenase-dependent mechanism. J. Clin. Invest. 118:3431-3439.

16. Cao, W., et al. 2008. Toll-like receptor-mediated induction of type I interferon in plasmacytoid dendritic cells requires the rapamycin-sensitive PI(3)K-mTOR-p70S6K pathway. Nat. Immunol. 9:1157-1164.

17. Flores-Villanueva, P.O., et al. 2001. Control of HIV-1 viremia and protection from AIDS are associated with HLA-Bw4 homozygosity. Proc. Natl. Acad. Sci. U. S. A. 98:5140-5145.

18. Hessell, A.J., et al. 2007. Fc receptor but not complement binding is important in antibody protection against HIV. Nature. 449:101-104.

19. Pereira, L.E., and Ansari, A.A. 2009. A case for innate immune effector mechanisms as contributors to disease resistance in SIV-infected sooty mangabeys. Curr. HIV Res. 7:12-22.

20. Raffatellu, M., et al. 2008. Simian immunodeficiency virus-induced mucosal interleukin-17 deficiency promotes Salmonella dissemination from the gut. Nat. Med. 14:421-428.

21. Quintana, F.J., et al. 2008. Control of T(reg) and $\mathrm{T}(\mathrm{H}) 17$ cell differentiation by the aryl hydrocarbon receptor. Nature. 453:65-71.

\title{
Phosducin - a candidate gene for stress- dependent hypertension
}

\author{
Guido Grassi \\ Clinica Medica, Dipartimento di Medicina Clinica, Prevenzione e Biotecnologie Sanitarie, \\ Università degli Studi di Milano-Bicocca, Ospedale San Gerardo Monza, Milan, Italy.
}

\begin{abstract}
Repeated exposure to stress may favor, both in experimental animals and in humans, an increase in blood pressure, leading in some instances to a true hypertensive state. It is thought that stress-induced hypertension is mediated by sympathetic nervous system activation that in turn, by exerting vasoconstrictor effects and increasing heart rate (and thus cardiac output), may promote the development and progression of the hypertensive state. A new study by Beetz and colleagues in this issue of the JCI, which reports the results of experimental studies carried out in both mice and humans, reveals the potential role of the phosducin gene in modulating the adrenergic and blood pressure responses to stress (see the related article beginning on page 3597 ).
\end{abstract}

Two major antecedents provide the background for the intriguing results provided by Beetz and coworkers in their study published in this issue of the JCI (1), which deals with the role of phosducin $(P D C)$ as

Conflict of interest: The author has declared that no conflict of interest exists.

Citation for this article: J. Clin. Invest. 119:3515-3518 (2009). doi:10.1172/JCI41508. a novel candidate gene for stress-dependent hypertension. The first antecedent dates back several years and is represented by evidence that stress may be involved in the pathogenesis of essential hypertension (also known as primary hypertension which, by definition, has no identified cause), a relationship that was first suggested by the pioneering studies of Cannon (2) and Folkow (3). These investigators, and later other distinguished scientists $(4,5)$, elegantly showed that emotional stress in experimental animals may trigger the so-called "defense reaction," also known as the "fight or flight response," which is mediated by increased activation of the sympathetic nervous system and characterized by an elevation in blood pressure, an increase in heart rate, and adrenergic stimulation together with marked vasodilatation in skeletal muscle. Many of the aforementioned cardiovascular responses to stress have also been described in humans. For example, more than 30 years ago Brod and coworkers (6) reported that a mental arithmetic task elicits a pressor and tachycardic response, similarly to what has been documented in response to other stressor stimuli such as the cold pressor test, the Stroop colorword conflict test, and the mirror imaging star-tracking test $(7,8)$. Key to the 